\title{
Plumbagin attenuates cancer cell growth and osteoclast formation in the bone microenvironment of mice
}

\author{
Wei YAN ${ }^{1,3, \#, ~ T i n g-y u ~ W A N G ~}{ }^{2, \#}$, Qi-ming FAN ${ }^{1}$, Lin $\mathrm{DU}^{1}$, Jia-ke XU ${ }^{4}$, Zan-jing ZHAI ${ }^{1}$, Hao-wei $\mathrm{LI}^{1}$, Ting-ting TANG ${ }^{1, *}$ \\ ${ }^{1}$ Shanghai Key Laboratory of Orthopaedic Implants, Department of Orthopaedic Surgery, Shanghai Ninth People's Hospital, Shanghai \\ Jiao Tong University School of Medicine, Shanghai 200011, China; ${ }^{2}$ Department of Pharmacy, Shanghai Ninth People's Hospital, \\ Shanghai Jiao Tong University School of Medicine, Shanghai 200011, China; ${ }^{3}$ Wendeng Zhenggu Hospital of Shandong Province, \\ Wendeng 264400, China; ${ }^{4}$ School of Pathology and Laboratory Medicine, University of Western Australia, Nedlands, 6009, Western \\ Australia, Australia
}

Aim: To investigate the effects of plumbagin, a naphthoquinone derived from the medicinal plant Plumbago zeylanica, on human breast cancer cell growth and the cancer cell-induced osteolysis in the bone microenvironment of mice.

Methods: Human breast cancer cell subline MDA-MB-231SA with the ability to spread and grow in the bone was tested. The cell proliferation was determined using the CCK-8 assay. Apoptosis was detected with Annexin V/PI double-labeled flow cytometry. Red fluorescent protein-labeled MDA-MB-231SArfp cells were injected into the right tibia of female BALB/c-nu/nu mice. Three days after the inoculation, the mice were injected with plumbagin $(2,4$, or $6 \mathrm{mg} / \mathrm{kg}$, ip) 5 times per week for 7 weeks. The growth of the tumor cells was monitored using an in vivo imaging system. After the mice were sacrificed, the hind limbs were removed for radiographic and histological analyses.

Results: Plumbagin (2.5-20 $\mu \mathrm{mol} / \mathrm{L})$ concentration-dependently inhibited the cell viability and induced apoptosis of MDA-MB-231SA cells in vitro (the $\mathrm{IC}_{50}$ value of inhibition of cell viability was $14.7 \mu \mathrm{mol} / \mathrm{L}$ ). Administration of plumbagin to breast cancer bearing mice delayed the tumor growth by $2-3$ weeks and reduced the tumor volume by $44 \%-74 \%$. The in vivo imaging study showed that plumbagin dose-dependently inhibited MDA-MB-231SArfp cell growth in bone microenvironment. Furthermore, X-ray images and micro-CT study demonstrated that plumbagin reduced bone erosion area and prevented a decrease in bone tissue volume. Histological studies showed that plumbagin dose-dependently inhibited the breast cancer cell growth, enhanced the cell apoptosis and reduced the number of TRAcP-positive osteoclasts.

Conclusion: Plumbagin inhibits the cell growth and induces apoptosis in human breast cancer cells in mice bone microenvironment, leading to significant reduction in osteolytic lesions caused by the tumor cells.

Keywords: plumbagin; naphthoquinone; breast cancer; lytic lesion; osteoclast; bone microenvironment; apoptosis; in vivo imaging

Acta Pharmacologica Sinica (2014) 35: 124-134; doi: 10.1038/aps.2013.152

\section{Introduction}

Bone tissues are the most frequent sites of metastases and are of particular clinical importance in breast cancer patients because of the prevalence of this disease; breast cancer is the second most common cancer in the world and the most commonly diagnosed cancer in women. Autopsy studies have demonstrated the presence of bone metastases in more than $70 \%$ of breast cancer patients ${ }^{[1,2]}$. Metastases are generally

\footnotetext{
\# These authors contributed equally to this work.

* To whom correspondence should be addressed.

E-mail ttt@sjtu.edu.cn

Received 2013-07-25 Accepted 2013-09-18
}

thought to cause many complications, including intractable bone pain, pathological fractures, hypercalcemia, nerve compression syndromes, and decreases in the quality of life ${ }^{[3]}$.

The development and outgrowth of these secondary lesions depend on the intricate cellular and molecular interactions between the breast tumor cells and the bone microenvironment. In particular, tumor cells can disrupt the bone homeostatic balance maintained by the two resident bone cell types, osteoclasts and osteoblasts, and this disruption has been shown to drive bone destruction and metastatic tumor growth $^{[2]}$. Tumor cells secrete signaling proteins, such as parathyroid hormone-related peptide $(\mathrm{PTHrP})^{[4]}$, to promote osteoclast differentiation and activity either directly or indirectly 
by altering the expression of receptor activator of nuclear factor- $\mathrm{KB}$ ligand (RANKL), an essential osteoclast differentiation cytokine, in osteoblasts. The resulting bone destruction releases a number of growth factors stored in the bone matrix, such as transforming growth factor- $\beta$ (TGF- $\beta$ ), which further stimulates the malignancy of the tumor cells and completes the so-called vicious cycle of bone metastasis. The current main drug treatment for skeletal lesions is the administration of bisphosphonates that block osteoclast activity; this treatment has been successful in slowing the progression of bone lesions but does not induce the regeneration of bone tissues or result in a cure ${ }^{[5]}$. Furthermore, a growing number of case reports have shown that long-term bisphosphonate therapy might result in osteonecrosis of the jaw $(\mathrm{ONJ})^{[6-9]}$.

In recent years, some natural compounds have been reported to have anticancer properties, such as cordycepin, which induces apoptosis and autophagy in breast cancer cells $^{[10]}$, and genistein, which inhibits the osteolytic bone metastasis of breast cancer and enhances the bone mineral levels in nude mice ${ }^{[11]}$. Resveratrol and sanguinarine were also shown to inhibit the proliferation and promote the apoptosis of osteosarcoma cells ${ }^{[12,13]}$. Plumbagin (5-hydroxy-2-methyl1,4-naphthoquinone), which is one of the most investigated compounds, is an analog of vitamin $\mathrm{K} 3$ that is derived from the roots of the medicinal plant Plumbago zeylanica, as well as the Droseraceae, Ancistrocladaceae and Dioncophyllaceae families. Plumbagin has been used as a hair dye and skin colorant and has also been used in the treatment of acne, ringworm, fungal, bacterial and viral infections and inflammatory diseases $^{[14]}$. Plumbagin has been safely used for centuries in Indian and Chinese medicine and exhibits anti-microbial properties ${ }^{[15]}$, anti-atherosclerotic effects ${ }^{[16]}$, anti-inflammatory effects $^{[17]}$, and anticancer activities both in vitro ${ }^{[18,19]}$ and in vivo ${ }^{[20,21]}$. Previous findings have suggested that plumbagin can inhibit cell proliferation and induce the apoptosis and autophagy of breast cancer cells in vitro ${ }^{[19]}$, as well as suppress the metastasis of prostate cancer ${ }^{[22]}$. One recent report also showed the effects of plumbagin on the suppression of osteoclast formation ${ }^{[23]}$. These data suggest that plumbagin might abrogate RANKL signaling and suppress osteoclastogenesis induced by breast cancer cells in mice. However, whether plumbagin can directly inhibit the growth of breast cancer cells in the bone microenvironment and reduce the cancerinduced bone destruction remain to be investigated. More information, such as the real-time monitoring of cancer cell growth and the dose-dependent effects of plumbagin in vivo, are needed to evaluate the potential uses of plumbagin in clinical applications.

The objective of this study was to determine the efficacy of different doses of plumbagin against the growth of breast cancer cells in the bone microenvironment in a well-characterized mouse model of breast cancer. We non-invasively and quantitatively monitored the growth of labeled tumor cells using an in vivo imaging system. Additionally, the osteolytic bone destruction caused by cancer cell growth was evaluated by $X$ ray, micro-CT, and histological observations. We believe that this systemic evaluation provides solid data regarding the potential use of plumbagin in the treatment of bone metastasis of breast cancer.

\section{Materials and methods Materials}

Plumbagin, dimethyl sulfoxide (DMSO), and thiazolyl blue tetrazolium bromide were purchased from Sigma-Aldrich (St Louis, MO, USA). For the cell culture experiments, plumbagin was dissolved in DMSO at a concentration of $200 \mathrm{mmol} / \mathrm{L}$ and was stored in a dark-colored bottle at $-20^{\circ} \mathrm{C}$. This stock solution was diluted further in cell culture medium immediately before use. For the animal experiments, plumbagin was dissolved in 5\% PEG 400 at the necessary concentrations.

\section{Cell culture}

The estrogen-independent human breast cancer cell subline MDA-MB-231SA was kindly provided by T YONEDA (University of Texas Health Science Centre at San Antonio, San Antonio, TX, USA). These cells were previously generated from MDA-MB-231 cells by the intracardiac inoculation and in vivo selection of cells that displayed the ability to spread and grow in the bone ${ }^{[24]}$. MDA-MB-231SArfp (RFP, red fluorescent protein) cells were generated by the stable transfection of the pDsRed2-N1 construct (Clontech, Mountain View, CA, USA $)^{[25]}$. The cell lines were cultured in Dulbecco's modified Eagle's medium (DMEM, Gibco, Invitrogen Ltd, Carlsbad, CA, USA) supplemented with 10\% fetal bovine serum (FBS), 1\% penicillin-streptomycin solution (Gibco, Invitrogen Ltd, Carlsbad, CA, USA), and $0.75 \mathrm{mg} / \mathrm{mL}$ G-418 (Invitrogen, Carlsbad, CA, USA) at $37^{\circ} \mathrm{C}$ in a humidified atmosphere with $5 \% \mathrm{CO}_{2}$.

\section{Cell viability assay}

Cell viability was assessed using the Cell Counting Kit-8 assay (CCK-8, Dojindo Laboratories, Kumamoto, Japan) according to previously reported methods ${ }^{[26]}$. Briefly, MDA-MB-231SArfp cells were grown overnight without treatment in 96-well plates (5000 cells/well). Next, the cells were incubated with a vehicle control (DMSO) or different concentrations of plumbagin (0-20 $\mathrm{mmol} / \mathrm{L})$ diluted from the $200 \mathrm{mmol} / \mathrm{L}$ stock. After a $48 \mathrm{~h}$ incubation, $10 \mu \mathrm{L}$ of the WST-8 reagent [2-(2-methoxy4-nitrophenyl)-3-(4-nitrophenyl)-5-(2,4-disulfonyl)-2H-tetrazolium] was added to each well, and the cells were incubated at $37^{\circ} \mathrm{C}$ for $3 \mathrm{~h}$. The optical densities (OD) of the wells were measured at $450 \mathrm{~nm}$ on a 96-well multi-scanner autoreader (Thermo Electron Corp, Waltham, MA, USA). Each condition was performed in eight replicate wells, and the total concentration of DMSO in the reaction mixtures never exceeded $0.1 \%$. The number of cells counted/OD value in the DMSO control wells was set to $100 \%$, and the number of cells in the plumbagin-treated cells was calculated relative to this control as the percentage of the surviving cells. At least three independent repeats of this experiment were performed.

\section{Apoptotic cell detection}

An Annexin-V-FITC kit (Bender Medsystems, Burlingame, 
CA, USA) was used to assess apoptosis according to the manufacturer's instructions. Briefly, the MDA-MB-231SArfp cells treated with DMSO (vehicle control) or different concentrations $(2.5,5.0$, or $7.5 \mu \mathrm{mol} / \mathrm{L})$ of plumbagin were resuspended in $500 \mu \mathrm{L}$ of binding buffer and were stained with AnnexinV-FITC and PI solution for $30 \mathrm{~min}$ at room temperature in the dark. The samples were analyzed immediately on a FACS Calibur flow cytometer (Becton, Dickinson and Co, San Jose, CA, USA).

\section{Mouse model and monitoring of breast cancer growth in bone}

Female BALB/c-nu/nu mice $(n=60), 4-5$ weeks of age, were obtained from the Shanghai Slac Laboratory Animal Company [Certificate No SCXK (Shanghai) 2007-0005]. The animals were maintained in plastic micro-isolator cages (5 mice/cage) under specific pathogen-free (SPF) conditions with a $12 \mathrm{~h}$ light/dark cycle at $22-24^{\circ} \mathrm{C}$ and $50 \%-55 \%$ humidity. Commercial mouse chow and water were provided. All animals were acclimatized for $7 \mathrm{~d}$ prior to their participation in the experiments. All mouse manipulations were performed within a laminar-flow hood under aseptic conditions; general anesthesia was used and consisted of ketamine administered by intraperitoneal (ip) injection $(0.2 \mathrm{~mL} / 100 \mathrm{~g}$ body weight) unless otherwise noted. According to the methods reported in previous studies ${ }^{[27,28]}, 50 \mu \mathrm{L}$ of MDA-MB-231SArfp cells at a concentration of $5 \times 10^{6}$ cells $/ \mathrm{mL}$ in phosphate-buffered saline (PBS) were injected intratibially into the anaesthetized nude mice on day 0 . The contralateral tibiae were similarly injected with PBS as a control. The mice were randomly assigned to four groups. Three days after cell implantation, the mice were treated with the vehicle $(100 \mu \mathrm{L}$ of $5 \%$ PEG, $n=10)$ or plumbagin $(2,4$, or $6 \mathrm{mg} / \mathrm{kg}$ body weight in the vehicle, $n=10$ per group) by ip injection five times per week for seven weeks.

Real-time non-invasive monitoring of MDA-MB-231SArfp breast cancer cell growth in the bone tissues was performed using the IVIS spectrum small-animal in vivo imaging system (Xenogen, Hopkinton, MA, USA), and the fluorescence intensity was determined in each mouse 1 week after the injection of the tumor cells. The tumor sizes were measured with calipers and recorded each week. Assuming an ellipsoid shape of the developing mass, the following formula was used to calculate the tumor volumes $(\mathrm{V}): \mathrm{V}=4 / 3 \Pi(\mathrm{a} / 2 \times b / 2 \times c / 2)$, where $a$ is the length, $b$ is the width, and $c$ is the height of the xenograft tumor.

\section{Evaluations of lytic lesions}

Mice were anaesthetized as described above and were examined for the development of osteolytic lesions by digital radiography (MX-50 desktop X-ray radiograph, Faxitron) 4 and 7 weeks after the injection of the tumor cells. At the end of the study, the mice were sacrificed, and specimens were harvested for micro-computed tomography (micro-CT) and histological evaluation. The bone erosion areas were determined using interactive image analysis software (ImageJ, NIH, USA) from the digital radiographs of the tibiae from the plumbagin- injected and vehicle-injected mice. Changes in the sizes of the osteolytic lesions were analyzed by comparing the radiographs taken at weeks 4 and 7 for each group of mice.

After the tissue harvest, representative micro-CT (SCANCO, $\mu \mathrm{CT} 80$ Switzerland) images of the tibiae were obtained. After the scans, reconstructions of the sections were performed with a modified Feldkamp cone-beam algorithm, with the beam hardening correction set to 50\%. The VGStudio MAX 1.2 software (Volume Graphics $\mathrm{GmbH}$ ) was used to obtain the threedimensional visualizations of the tibiae from the reconstructed sections, and a $4 \mathrm{~mm}$ area below the tibial plateau was selected to calculate the bone volume fractions (bone volume/total volume, BV/TV). All of the experimental protocols were approved by the Animal Ethics Committee of the Shanghai Jiaotong University School of Medicine (Shanghai, China).

\section{H\&E staining and RFP immunohistochemistry}

The harvested tibiae were fixed for $36 \mathrm{~h}$ in $4 \%$ paraformaldehyde buffered with $0.1 \mathrm{~mol} / \mathrm{L}$ phosphate buffer ( $\mathrm{pH} 7.4$ ) and were decalcified in $10 \%$ EDTA at $4^{\circ} \mathrm{C}$ for three weeks. Paraffin sections were made according to the standard conventional methods and were stained with H\&E.

Serial sections ( $5 \mu \mathrm{m}$ thick) were mounted on glass slides coated with $10 \%$ polylysine. The sections were deparaffinized with xylene and rehydrated in graded concentrations of ethanol. Antigen retrieval was performed in a protease $\mathrm{K}$ solution at $37^{\circ} \mathrm{C}$ for $20-25 \mathrm{~min}$. The endogenous peroxidase activity was blocked by immersion in $0.3 \%$ methanolic peroxide for $40 \mathrm{~min}$. The immunoreactivity of the sections was enhanced by microwaving the tissue sections for $10 \mathrm{~min}$ in $0.1 \mathrm{~mol} / \mathrm{L}$ citrate buffer. Next, the sections were incubated with a rabbit polyclonal anti-RFP primary antibody at $4{ }^{\circ} \mathrm{C}$ overnight. After washing, the slides were incubated with a biotinylated or fluorogenic secondary antibody (polyclonal goat anti-rabbit immunoglobulin). After washing, the sections were incubated with an avidin-biotin-peroxidase complex. The RFP activity was visualized with 3,3'-diaminobenzidine or 4',6-diamidino2-phenylindole (DAPI, $10 \mathrm{ng} / \mathrm{mL}$ ) for $10 \mathrm{~min}$. The cells were analyzed by microscopy.

\section{Ki67 immunohistochemistry and TUNEL staining}

As an indicator of proliferation, sections $(5 \mu \mathrm{m})$ were examined immunohistochemically for the expression of human Ki67 with a polyclonal rabbit antibody against human Ki67 (Santa Cruz Biotechnology Inc, USA) and a secondary goat anti-rabbit antibody (Vector Laboratories, Burlingame, CA, USA). Tumor cell apoptosis rates were assessed using the transferase-mediated dUTP biotin nick end-labeling (TUNEL) assay, which was performed on the $5-\mu \mathrm{m}$ sections with the In Situ Cell Death Detection Kit, POD (Roche Diagnostics) according to the manufacturer's protocol. The proportion of Ki67-positive cells and the apoptotic TUNEL-stained cells was determined by counting the positive and negative cells in five random fields of the non-necrotic areas of the tumors in a representative section of each bone specimen. 
Staining for tartrate-resistant acid phosphatase (TRAcP)

Paraffin sections were made according to the standard conventional methods and were stained with tartrate-resistant acid phosphatase (TRAcP), a osteoclast marker, using a commercial kit (387A-1KT, Sigma-Aldrich) according to the manufacturer's protocol. Briefly, the sections were fixed for a short time in buffered acetone, followed by incubation in a solution of naphthol AS-BI phosphoric acid, freshly diazotized fast garnet GBC, acetate buffer, and tartrate buffer for $30 \mathrm{~min}$ at $37^{\circ} \mathrm{C}$. Following a rinse in deionized water, the sections were airdried and mounted with neutral resin. Osteoclasts were identified as TRAcP-positive multinucleated cells associated with the bone surfaces. The number of osteoclasts at the interface between the tumor and the bone was counted in five fields per section (x400 magnification), and the osteoclast number per $\mathrm{mm}$ of the tumor bone interface was calculated.

\section{Statistical analysis}

The data are presented as the mean \pm SD. A Student's $t$-test was used to determine the significance between the groups. $P$ values of less than 0.05 were considered significant. All statistical analyses were performed with the SPSS (Statistical Package for the Social Sciences) 13.0 software.
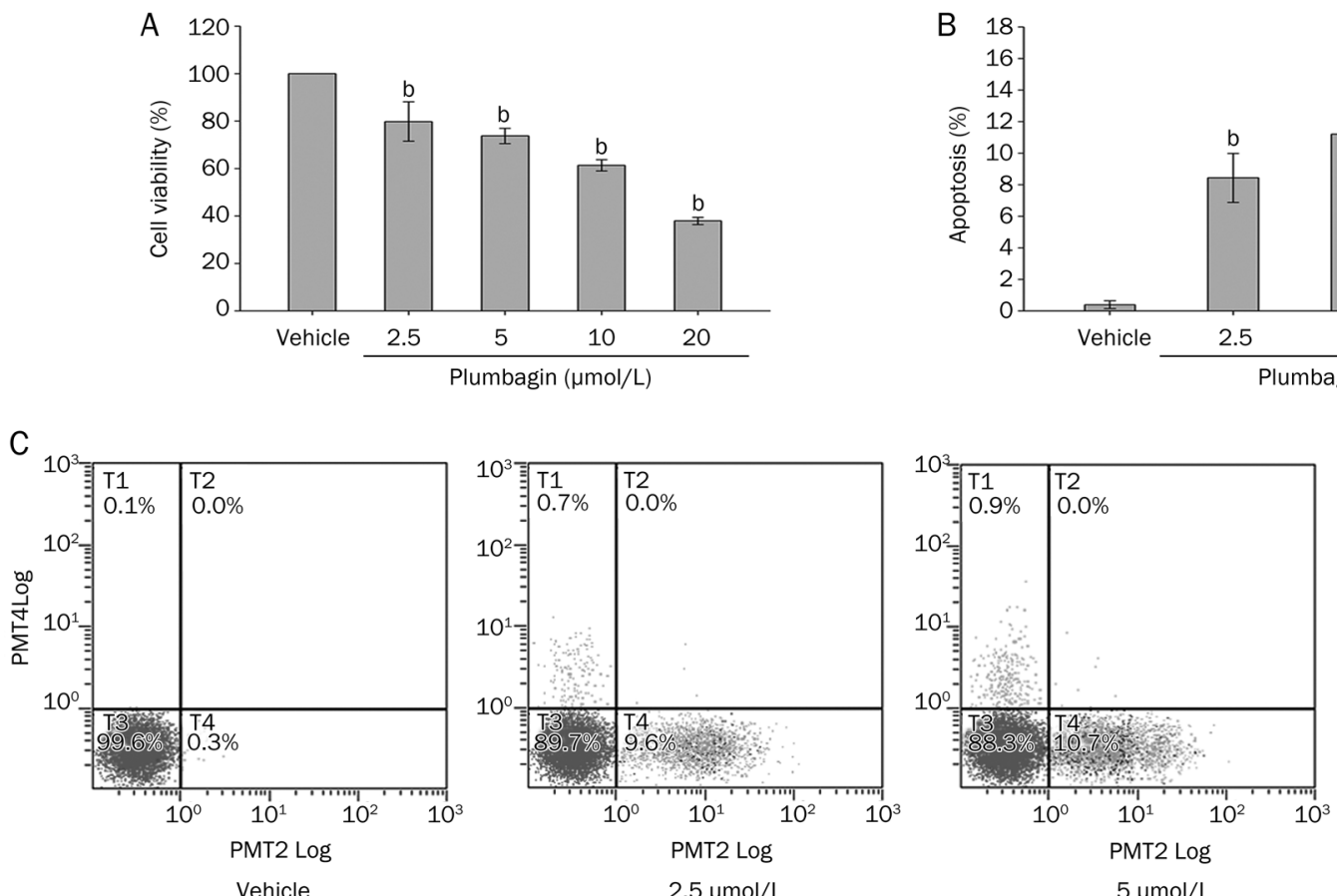

$5 \mu \mathrm{mol} / \mathrm{L}$

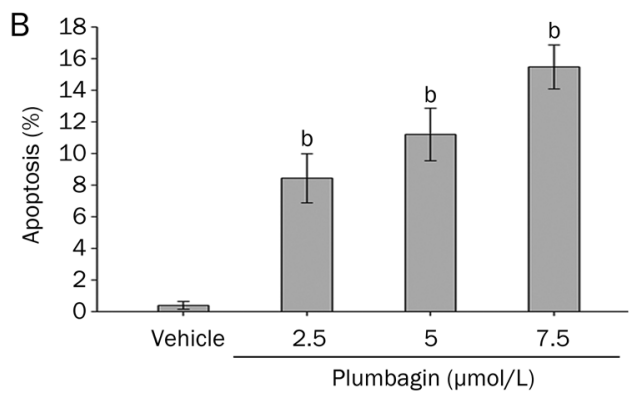

\section{Results}

Plumbagin decreased the viability and enhanced the apoptosis of breast cancer cells

Cellular viability was assayed by treating MDA-MB-231SArfp cells with various concentrations of plumbagin, followed by analysis with the CCK-8 viability assay. We observed that cellular viability was suppressed by plumbagin in a dosedependent manner in the breast cancer cells (Figure 1A). The $\mathrm{IC}_{50}$ value of plumbagin in the MDA-MB-231SArfp cells was $14.7 \mu \mathrm{mol} / \mathrm{L}$.

The amount of apoptotic cell death was quantified using Annexin V-FITC/PI double-labeled flow cytometry. The MDA-MB-231SArfp cells were pretreated with varying concentrations of plumbagin, which led to increases in apoptosis (Figure 1C). The total apoptosis rates were $0.41 \% \pm 0.25 \%$, $8.43 \% \pm 1.55 \%, 11.20 \% \pm 1.66 \%$, and $15.47 \% \pm 1.39 \%$ for plumbagin concentrations of $0,2.5,5$ and $7.5 \mu \mathrm{mol} / \mathrm{L}$, respectively (Figure 1B).

Plumbagin inhibits the growth of MDA-MB-231SArfp cells in the bone environment

In the animal experiments, plumbagin $(2,4$, or $6 \mathrm{mg} / \mathrm{kg}$ body weight) was administered by intraperitoneal injection three

Plumbagin

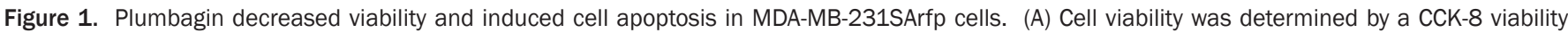

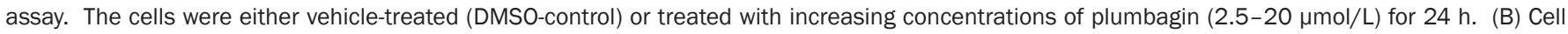

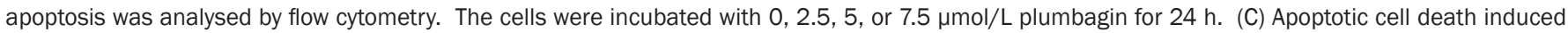

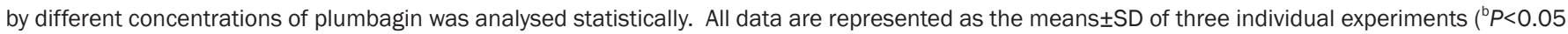
compared with the vehicle-treated group). 
A
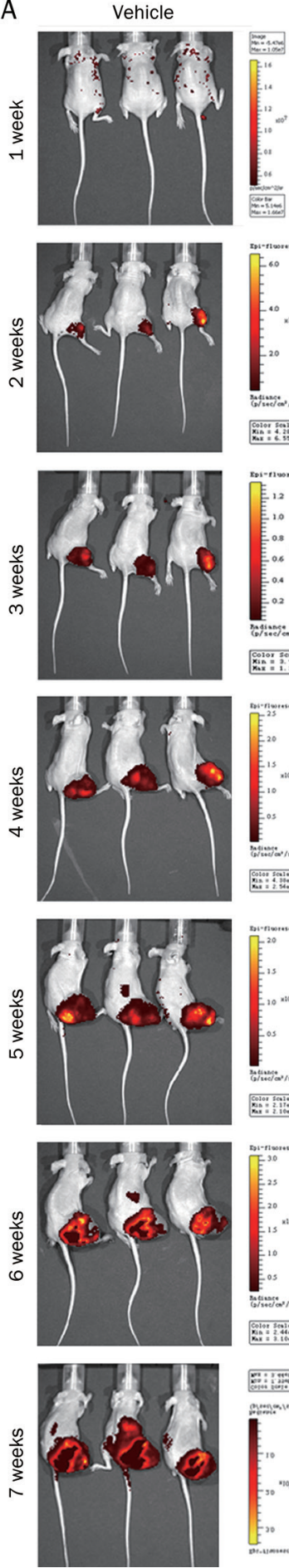

PL-2 mg
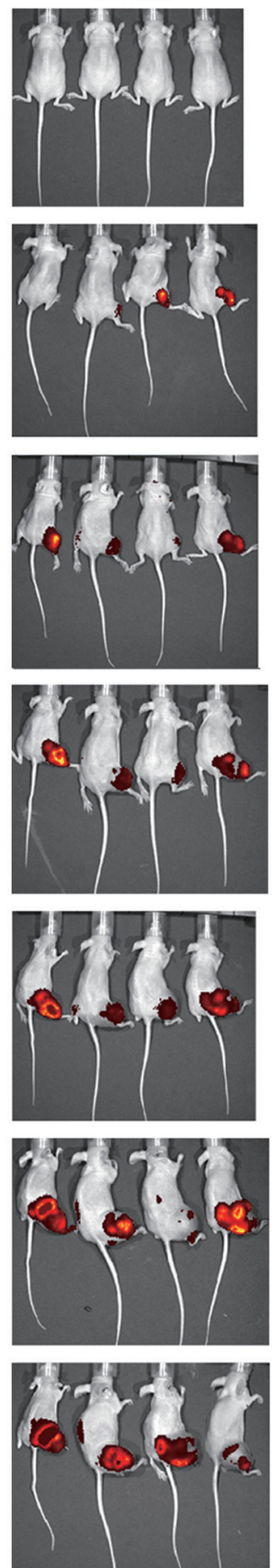
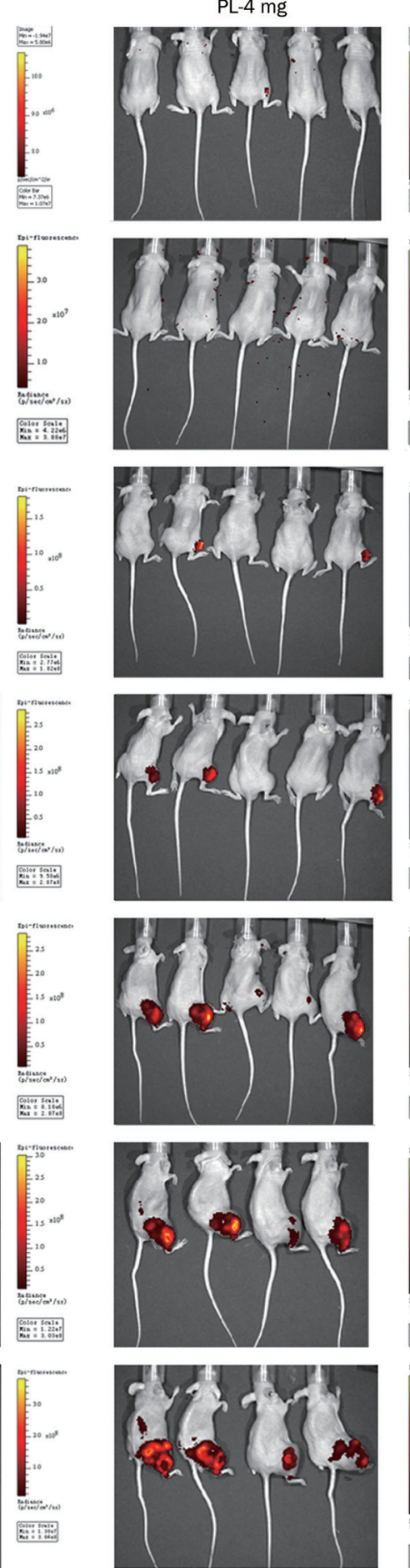
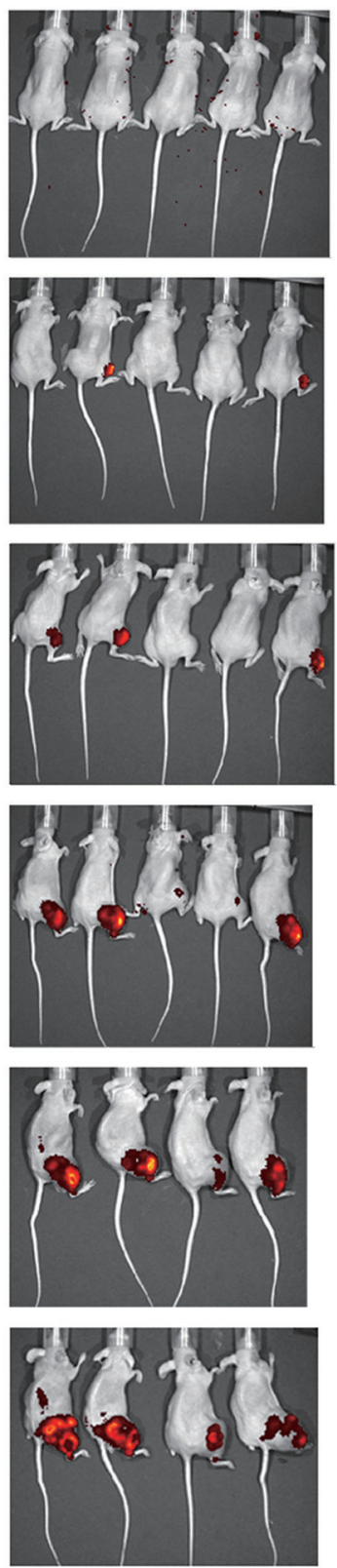
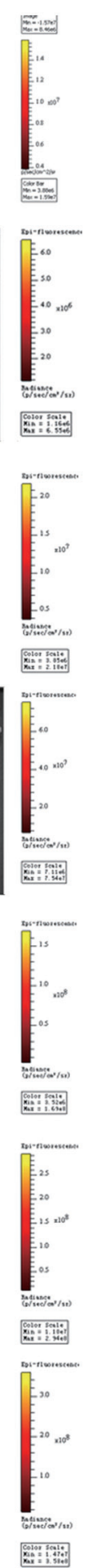

PL-6 mg
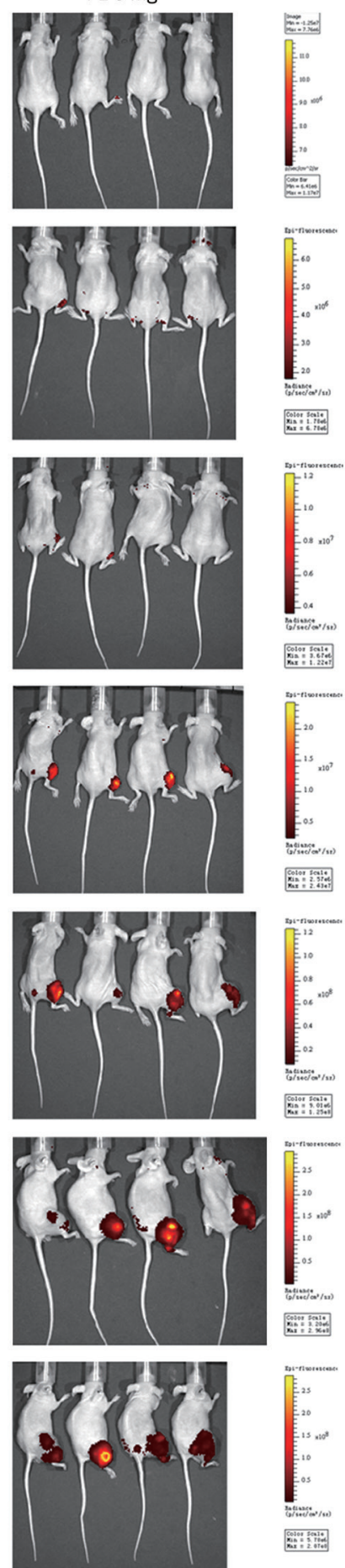

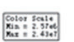

Figure 2A. Plumbagin inhibits the growth of MDA-MB-231SArfp cells in athymic nude mice. The animals were treated with plumbagin (2, 4, or 6 mg/kg of body weight) or vehicle (5\% PEG) only for five days per week by ip injection beginning at three days post-cell implantation. (A) Non-invasive monitoring of cell growth with the in vivo imaging system each week post-cell implantation.

days after MDA-MB-231 cells were intra-tibially injected. The in vivo imaging system showed that plumbagin administration decreased the fluorescence intensities of the tumors in a dose-dependent manner compared to the vehicle-treated group (Figure 2A and 2B). Plumbagin treatment delayed tumor growth by 2-3 weeks and reduced the tumor volumes by $44 \%-74 \%$ (Figure 2C). A correlation was observed between the tumor volume and fluorescence intensity (Figure 2D). At week 6, one mouse from the PL-4 mg-treated group died due to an injection accident. The plumbagin-treated mice gained 

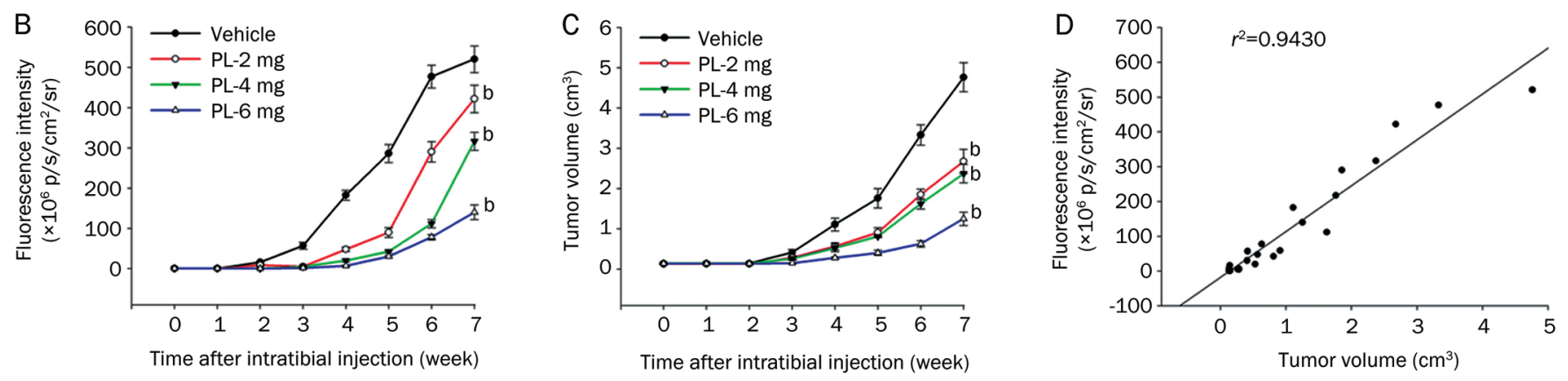

Figure 2B-2D. (B) The determined fluorescence intensity indicated changes in cell growth in the bone microenvironment. (C) Tumour growth was measured weekly with digital calipers. (D) The correlation between tumour volume and fluorescence intensity $\left(r^{2}=0.9430 ;{ }^{b} P<0.05\right.$ compared with the vehicle-treated group).
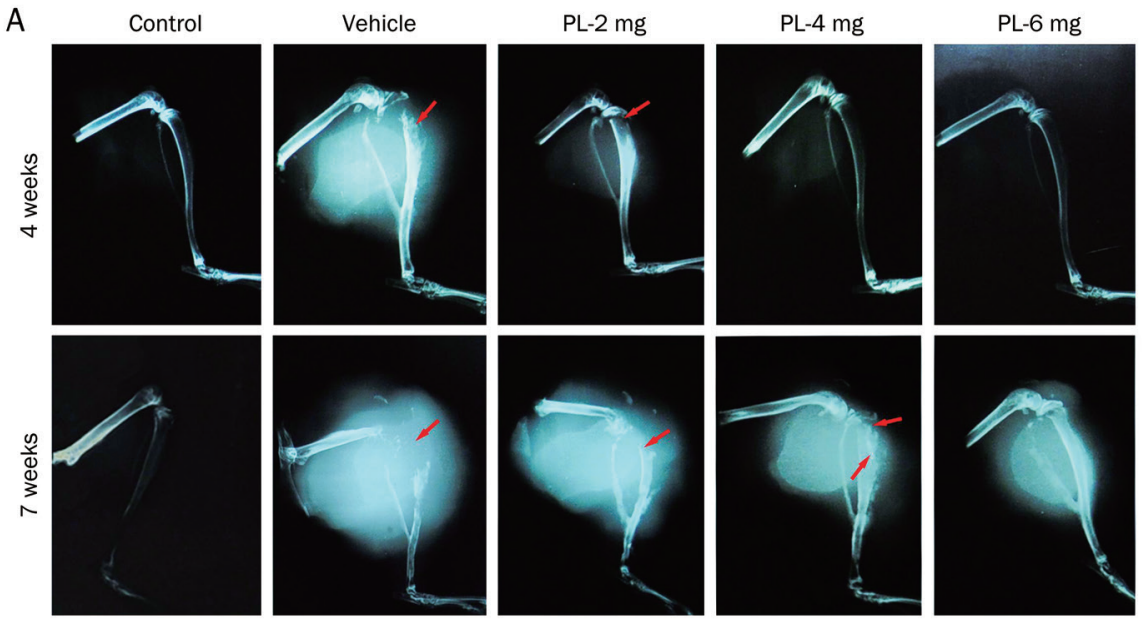

D

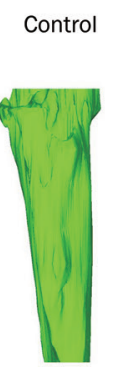

PL-2 mg

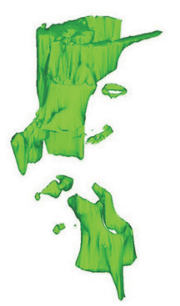

PL-4 mg

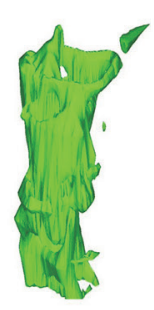

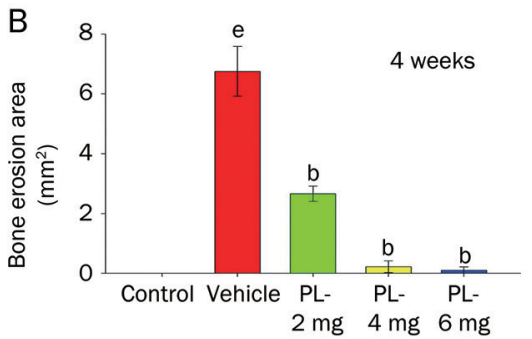
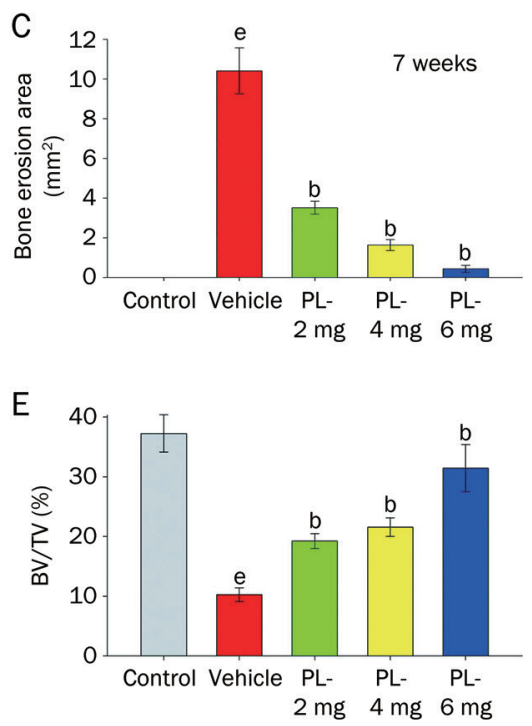

Figure 3. Plumbagin suppressed the osteolysis induced by MDA-MB-231SArfp breast cancer cells in tumour-bearing mice. The mice were treated with vehicle (5\% PEG) or plumbagin (2, 4, or $6 \mathrm{mg} / \mathrm{kg}$ of body weight) five times per week. (A) Representative radiographs of osteolytic lesions in the tibiae of tumour-bearing mice at weeks 4 and 7. (B, C) The dose related effects of plumbagin on osteolytic bone lesions at weeks 4 (B) or 7 (C). (D) Representative micro-CT images of the tibiae of tumour-bearing mice at week 7. (E) Plumbagin treatment prevented the decrease of the trabecular/ cancellous bone volume (BV/TV, bone volume/total volume) in the tibial platforms of tumour-bearing mice $\left({ }^{\mathrm{b}} P<0.05\right.$ compared with the vehicle-treated group. ${ }^{\mathrm{e}} P<0.05$ compared with the control group).

weight and exhibited no obvious toxic effects.

\section{Plumbagin suppresses osteolysis in the tumor-bearing mice}

To identify the osteolytic bone destruction, we analyzed osteolytic lesions using radiography and micro-CT analysis. Four weeks after tumor cell implantation, lytic bone lesions were established in $100 \%$ of the animals from the control and 2 $\mathrm{mg} / \mathrm{kg}$ plumbagin-treated groups, as determined by standard X-rays (Figure 3A). During the following three weeks, the areas of bone erosion at least doubled in the vehicle-treated 
mice. In contrast, in the mice treated with plumbagin, the expansion of the osteolytic lesions was significantly arrested in the bone erosion areas at week 7 (Figure 3C) compared to week 4 (Figure 3B). Lytic lesions were not observed in any of the sham-injected legs at any of the time points. Plumbagin reduced the bone erosion areas, as shown in the representative radiographs in Figures 3A and 3D, and the increased bone volume (Figure 3E).

Histologic demonstration of reduced osteolytic lesions and arrested cancer cell growth

After the tissue was harvested and fixed, 5- $\mu \mathrm{m}$ sections were cut from each specimen and stained with H\&E for routine histological examinations. In the sections from the vehicletreated mice, almost all of the metaphyseal bone had disappeared, and the joints were severely destroyed. In contrast, in the sections from the plumbagin-treated mice, nearly intact joints and some metaphyseal bone were observed; this suggested that plumbagin effectively reduced the bone destruction caused by the cancer cells when administered at doses of 2-6 mg/kg over 7 weeks (Figure 4A). The RFP immunostain- ing (immunohistochemistry in Figure 4B and immunofluorescence in Figure $4 \mathrm{C}$ ) also demonstrated the growth of the MDA-MB-231SArfp cells in the bone microenvironment.

Immunohistochemistry analysis showed that plumbagin decreased the number of Ki67-positive tumor cells (Figure $5 \mathrm{~A}, 5 \mathrm{~B}$, and 5D) and increased the number of TUNEL-positive tumor cells (Figure 5C and 5E) in the sections; these data indicate the decreased proliferation (vehicle, $34.0 \%$; plumbagin (PL)-2 mg, 18.5\%; PL-4 mg, 11.0\%; PL-6 mg, 5.5\%; P<0.05 vs vehicle) and the increased apoptosis (vehicle, 1.2\%; PL-2 mg, $3.2 \%$; PL-4 mg, 5.8\%; PL-6 mg, 13.1\%; P<0.05 vs vehicle) of the MDA-MB-231SArfp cells in the bone microenvironments of the plumbagin-treated mice.

\section{Decreased osteoclast numbers in the plumbagin-treated tumor-} bearing mice

Osteoclasts were identified as TRAcP-positive multinucleated cells that were associated with the bone surfaces. The number of osteoclasts at the interface between the tumor and bone were counted in five fields per section $(\times 400$ magnification), and the osteoclast numbers per mm of tumor bone inter-
A
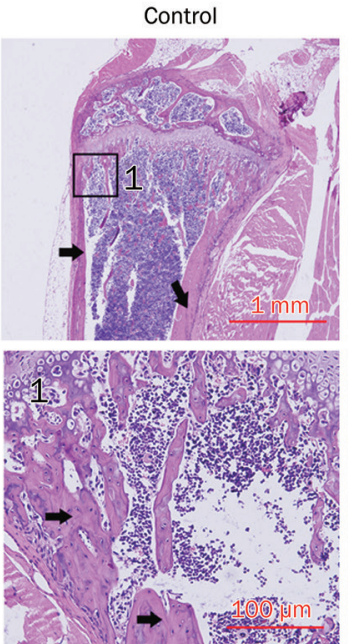

B

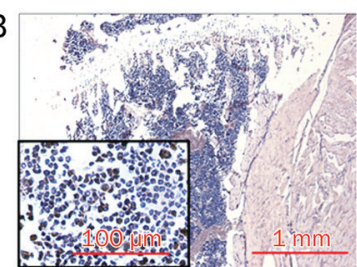

C

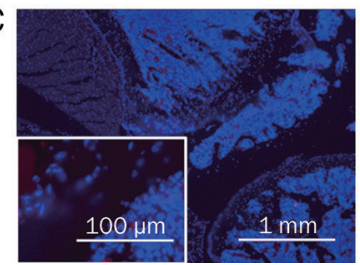

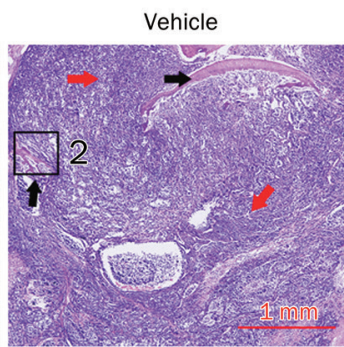
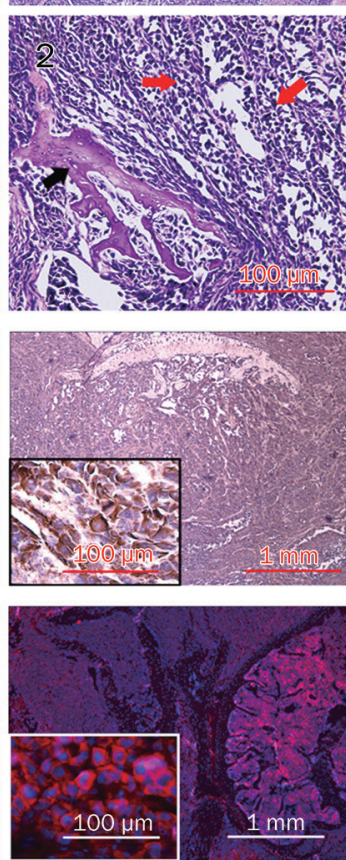
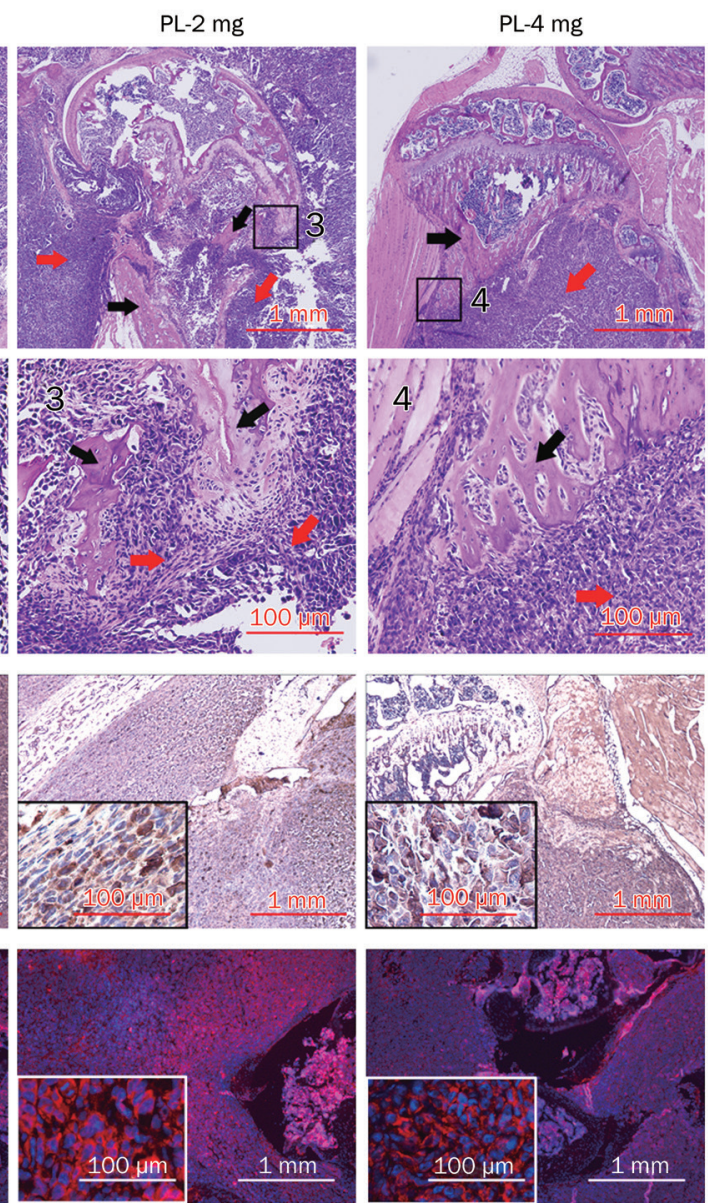
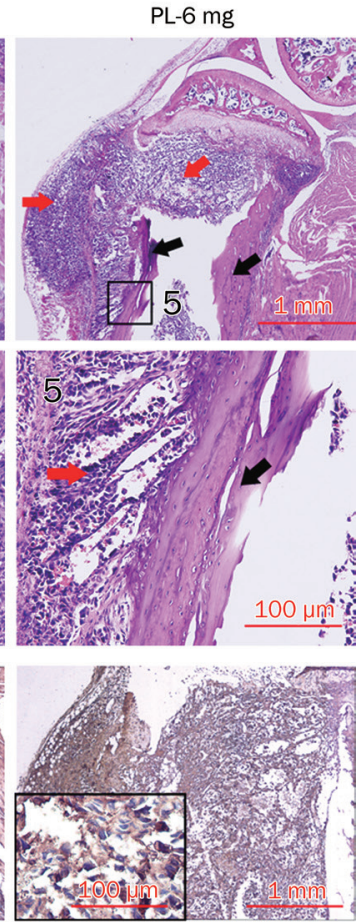

Figure 4. Representative histological and immunostaining images at week 7 indicated that plumbagin reduced the osteolytic lesions and arrested the growth of cancer cells in bone tissue. (A) H\&E staining micrographs of metaphyseal bone in the tibiae. (B) RFP immunohistochemistry of tumour tissues with DAB coloration. (C) Immunofluorescence analysis of tumour tissues (nuclei, blue; RFP, red). Data in B and C demonstrate that the growth of MDA-MB-231SArfp cells in the bone microenvironment was arrested by plumbagin. 

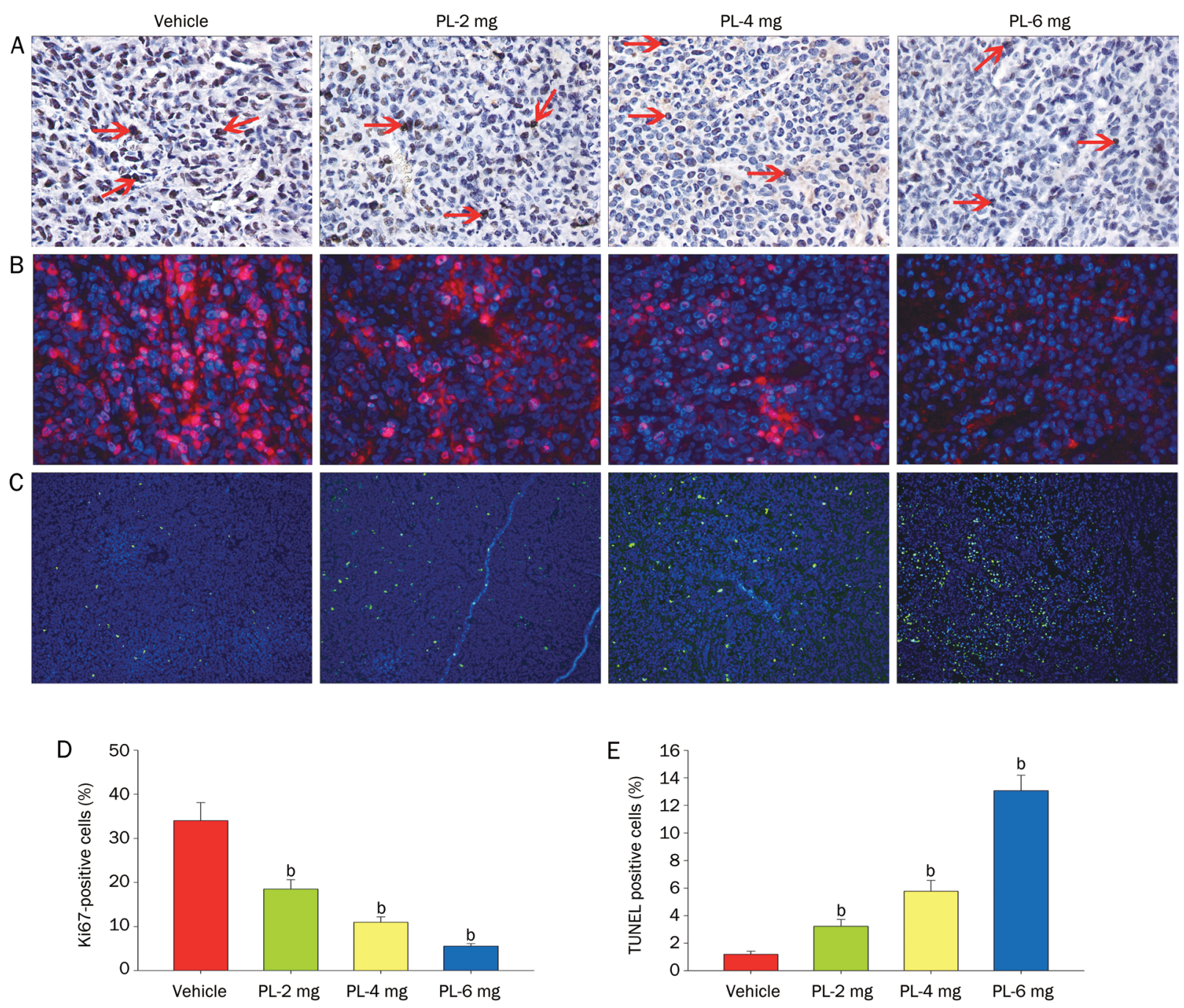

Figure 5. Histological demonstration of the proliferation and apoptosis of MDA-MB-231SArfp breast cancer cells in bone tissue. The mice were treated with vehicle (5\% PEG) or plumbagin $(2,4$, or $6 \mathrm{mg} / \mathrm{kg}$ of body weight) five times per week. (A) Immunohistochemical staining of tumour tissues; red arrows indicate Ki67-positive proliferating cancer cells (magnification, $\times 200$ ). (B) Immunofluorescent staining of tumour tissues; red staining indicates Ki67-positive proliferating cancer cells (magnification, $\times 200$ ). (C) TUNEL staining of tumour tissues indicate the apoptosis of cancer cells (green, TUNEL; magnification, $\times 40$ ). (D, E) Histomorphometric quantifications of proliferating (D) and apoptotic (E) cells in the bone microenvironment in mice. All data are expressed as the mean $\pm \mathrm{SD}$ (bars) $\left({ }^{\mathrm{b}} \mathrm{P}<0.05\right.$ compared with the vehicle-treated group).

face were also calculated. Plumbagin treatment significantly reduced the number of osteoclasts at the interface between the tumor and bone, and osteoclast activity was largely inhibited (Figure 6A and 6B).

\section{Discussion}

Breast cancer is the most common human neoplasm in both developed and developing countries ${ }^{[19]}$, and bone metastasis is commonly associated with breast cancer ${ }^{[23]}$. In our study, we found that plumbagin effectively inhibited tumor cell growth in vitro concomitant with the induction of cell apoptosis; furthermore, plumbagin inhibited tumor cell growth in nude mice and suppressed the osteolysis induced by the MDA-MB231SArfp breast cancer cells.

The results described herein clearly suggest that plumbagin inhibits cell growth and viability and leads to the induction of apoptosis in breast cancer cells. We observed the induction of apoptosis in the triple-negative breast cancer cell line MDAMB-231, against which there is no effective targeted therapy ${ }^{[29]}$. Ki67 immunohistochemical staining and TUNEL staining of the tissue sections from the plumbagin-treated mice confirmed the apoptosis of the cancer cells in the bone microenvironment. Ordinary chemotherapy for the treatment of breast cancer has strong side effects, and we speculated that plumbagin treatment might be associated with better prognosis and survival rates in breast cancer patients based on its ability to induce apoptosis selectively in cancer cells.

Although we did not investigate the mechanism of the plumbagin-induced apoptosis in this paper, some previous reports have investigated the involved signaling pathways. 
A
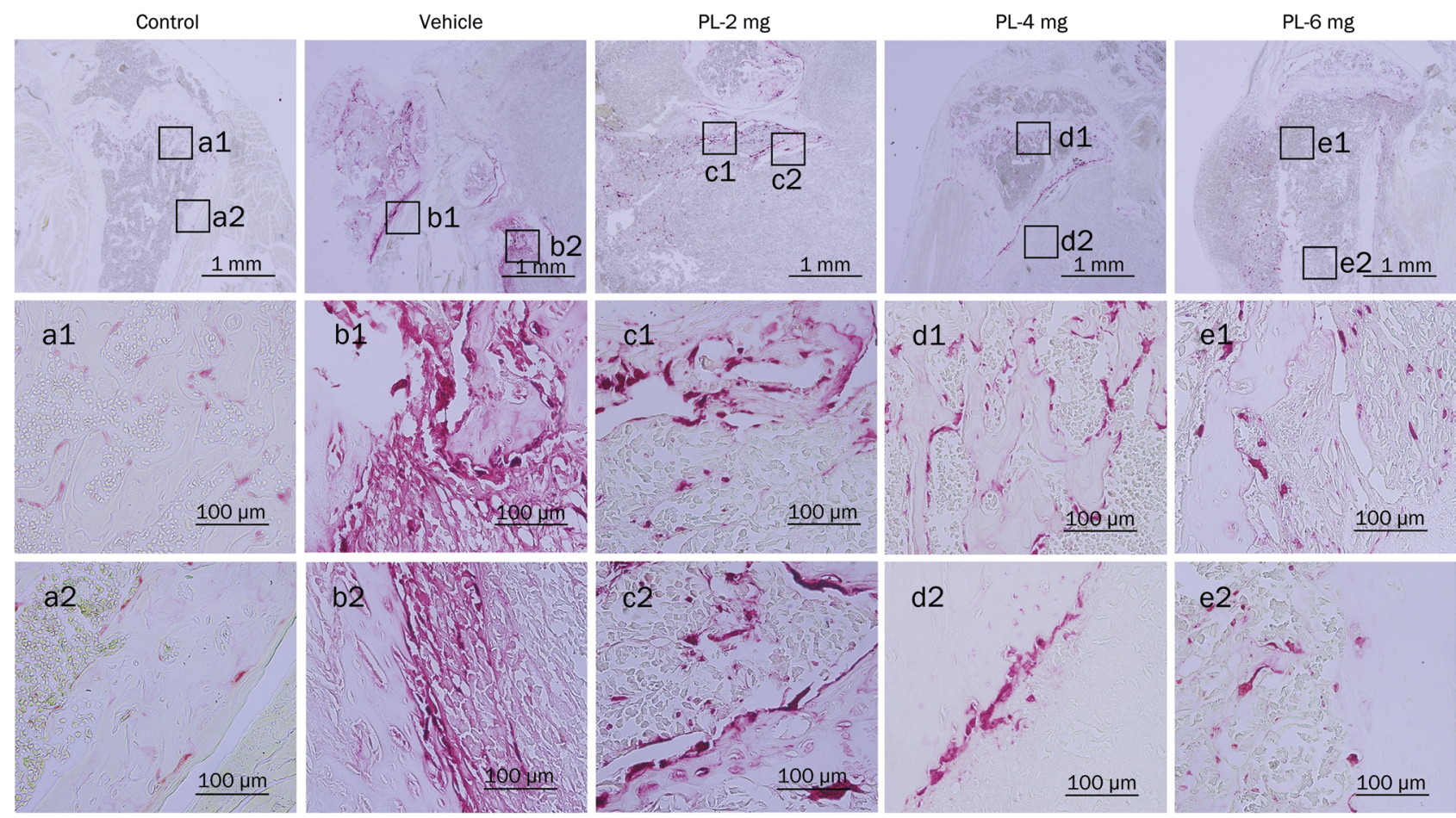
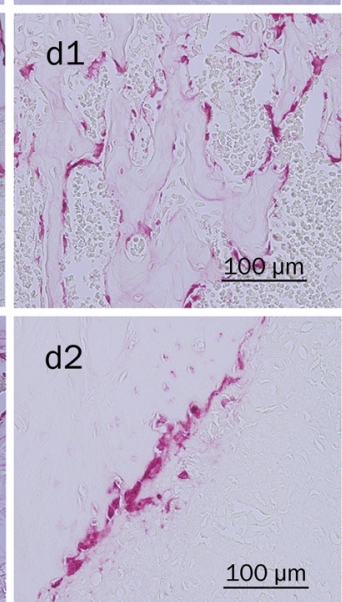
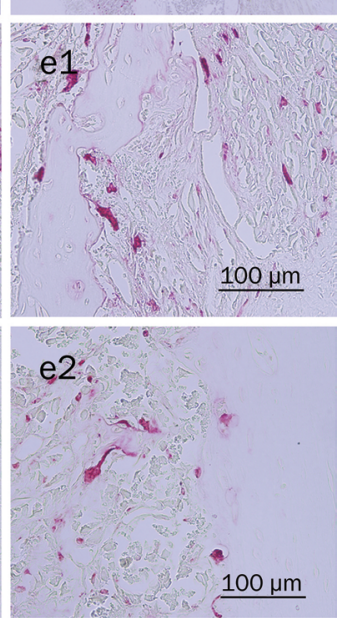

B

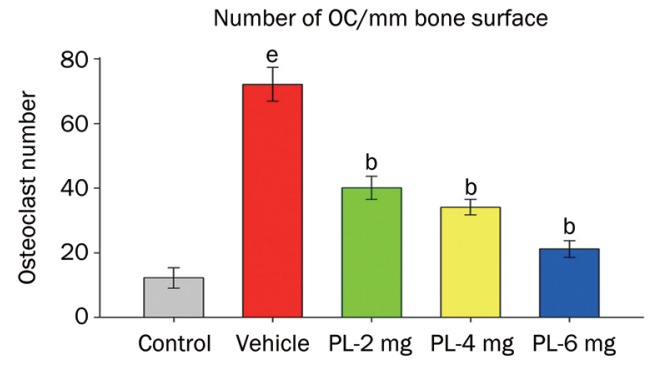

One previous study found that plumbagin predominantly triggered autophagic cell death but not apoptosis and that plumbagin induced a $\mathrm{G}_{2}-\mathrm{M}$ arrest and autophagy by inhibiting the AKT/mammalian target of rapamycin pathway in breast cancer cells ${ }^{[19]}$. Interestingly, another report demonstrated that plumbagin significantly inhibited the growth of breast cancer cells but had no effects on normal breast epithelial cells ${ }^{[30]}$. Using ER-positive MCF-7 and ER-negative MDA-MB-231 breast cancer cells, the researchers found that plumbagininduced apoptosis occurred concomitantly with the inactivation of Bcl-2 and a decrease in the DNA binding activity of NF- $\kappa B$. A recent study examined the anticancer potential of plumbagin against Her2-overexpressing breast cancer cells and found that the antiproliferative activity of plumbagin was associated with apoptotic cell death, as determined by caspase activation and an increase in the sub- $\mathrm{G}_{1}$ fraction of the cell cycle ${ }^{[31]}$. These data support our findings and provide insight into the mechanisms of plumbagin-induced apoptosis. Interestingly, Aziz and colleagues demonstrated the apoptosis-inducing effects of plumbagin in prostate cancer cells, regardless of hormone-responsiveness ${ }^{[32]}$; recently, the same
Figure 6. TRACP staining indicated that plumbagin inhibited osteoclast activity in tumour-bearing bones. (A) TRAcP stain-positive osteoclasts (purple-red) in the tibiae of mice treated with vehicle or plumbagin (2, 4, or $6 \mathrm{mg} / \mathrm{kg}$ of body weight). (B) Histomorphometric quantification of the active osteoclasts at the eroded bone surfaces. All data are expressed as the mean \pm SD $\left({ }^{b} P<0.05\right.$ compared with the vehicle-treated group; ${ }^{e} P<0.05$ compared with the control group).

group reported that plumbagin inhibited the diffusion of prostate cancer and the progression to poorly differentiated carcinoma in the TRAMP prostate cancer mouse model ${ }^{[33]}$, further suggesting that plumbagin might be an effective agent against cancer cells, regardless of the differences in the expression status of important target genes.

Most studies have reported plumbagin treatment doses of 2 $\mathrm{mg} / \mathrm{kg}$ body weight in tumor-burdened nude mice and have not investigated the dose-related effects ${ }^{[19]}$. However, an ear lier study found that the acute $\mathrm{LD}_{50}$ of plumbagin in normal mice was $9.4 \mathrm{mg} / \mathrm{kg}$ body weight and that single doses of plumbagin below $7 \mathrm{mg} / \mathrm{kg}$ were tolerated by the mice without any acute side effects. Furthermore, plumbagin doses of $5 \mathrm{mg} / \mathrm{kg}$ did not result in mortality within $14 \mathrm{~d}$, and single doses of 2-6 mg/ $\mathrm{kg}$ administered ip inhibited exponential tumor growth ${ }^{[34]}$. Thus, we treated the tumor-bearing mice with three doses of plumbagin $(2,4$, or $6 \mathrm{mg} / \mathrm{kg})$ that were below the reported $\mathrm{LD}_{50}$ of plumbagin to determine whether the increased doses had improved effects. We found that as the plumbagin dose increased, the inhibitory effects on tumor growth, bone damage, and osteoclast activity were improved 
significantly, which provides a reference for the potential clinical applications of plumbagin.

In this paper, we used an IVIS in vivo imaging system to non-invasively and intuitively monitor the weekly growth of the tumors in the bone microenvironment. This system quantified the number of tumor cells based on the fluorescence intensity of the labeled cells, which correlated well with the measured tumor volumes. Based on the determined realtime cell growth curves, we demonstrated that plumbagin could directly inhibit the growth of breast cancer cells in the bone microenvironment. The data from the X-ray, micro-CT, and histological analyses also significantly demonstrated the reduced osteolytic lesions in the plumbagin-treated mice compared to the vehicle-treated mice. Recently, one study showed that plumbagin could inhibit bone metastases of breast cancer by modulating the tumor-bone microenvironment. The researchers suggested that plumbagin could abrogate the RANKL-induced NF-KB and MAPK pathways by blocking the association of RANK and TRAF6 during osteoclastogenesis and inhibit the cancer cell induced-osteoclastogenesis, as well as the secretion of osteoclast-activating factors, such as IL-1 $\beta$ and IL-6 in vivo ${ }^{[35]}$.

In conclusion, the data reported herein, along with the data reported previously by other investigators, shows that plumbagin might be an effective therapeutic agent against cancer cells. After combining data from the in vivo imaging system, $\mathrm{X}$-ray, micro-CT, histology, and immunohistochemical analyses, we confirmed that plumbagin inhibited cell growth and enhanced apoptosis in human breast cancer cells, as well as inhibited the bone resorption induced by the tumor cells. Our data provide a solid basis for the future therapeutic application of plumbagin in the treatment of breast cancer bone metastases.

\section{Acknowledgements}

This work was supported by the grants from the National Natural Science Foundation of China (№ 81172549, 81228013 and 81301531), the Shanghai Science and Technology Development Fund (№ 11XD1403300 and 12140901300), the Program of Key Disciplines of Shanghai Municipal Education Commission (№ J50206), and the Opening Project of Shanghai Key Laboratory of Orthopaedic Implant (KFKT2011003). The authors would like to thank Dr John PRICE for providing the MDA-MB231SArfp cell line.

\section{Author contribution}

Wei YAN, Ting-yu WANG, Jia-ke XU, and Ting-ting TANG designed the experiments; Wei YAN, Ting-yu WANG, Lin DU, Zan-jing ZHAI, and Hao-wei LI performed the research; Wei YAN, Ting-yu WANG, Qi-ming FAN, and Ting-ting TANG analyzed the data; Wei YAN, Ting-yu WANG, and Ting-ting TANG wrote the paper.

\section{References}

1 Kamangar F, Dores GM, Anderson WF. Patterns of cancer incidence, mortality, and prevalence across five continents: defining priorities to reduce cancer disparities in different geographic regions of the world. J Clin Oncol 2006; 24: 2137-50.

2 Mundy GR. Metastasis to bone: causes, consequences and therapeutic opportunities. Nat Rev Cancer 2002; 2: 584-93.

3 Rose A, Siegel PM. Breast cancer-derived factors facilitate osteolytic bone metastasis. Bull Cancer 2006; 93: 931-43.

4 Guise TA, Yin JJ, Taylor S, Kumagai Y, Dallas M, Boyce B, et al. Evidence for a causal role of parathyroid hormone-related protein in the pathogenesis of human breast cancer-mediated osteolysis. J Clin Invest 1996; 98: 1544.

5 Lipton A, Theriault RL, Hortobagyi GN, Simeone J, Knight RD, Mellars $\mathrm{K}$, et al. Pamidronate prevents skeletal complications and is effective palliative treatment in women with breast carcinoma and osteolytic bone metastases. Cancer 2000; 88: 1082-90.

6 Bamias A, Kastritis E, Bamia C, Moulopoulos LA, Melakopoulos I, Bozas G, et al. Osteonecrosis of the jaw in cancer after treatment with bisphosphonates: incidence and risk factors. J Clin Oncol 2005; 23: 8580-7.

7 Dimopoulos MA, Kastritis E, Anagnostopoulos A, Melakopoulos I, Gika D, Moulopoulos LA, et al. Osteonecrosis of the jaw in patients with multiple myeloma treated with bisphosphonates: evidence of increased risk after treatment with zoledronic acid. Haematologica 2006; 91: 968-71.

8 Durie BGM, Katz M, Crowley J, Woo SB, Hande K, Richardson PG, et al. Osteonecrosis of the jaw and bisphosphonates. New Engl J Med 2005; 353: 99-102.

9 Hoff AO, Toth BB, Altundag K, Johnson MM, Warneke CL, Hu M, et al. Frequency and risk factors associated with osteonecrosis of the jaw in cancer patients treated with intravenous bisphosphonates. J Bone Mineral Res 2008; 23: 826-36.

10 Choi S, Lim MH, Kim KM, Jeon BH, Song WO, Kim TW. Cordycepininduced apoptosis and autophagy in breast cancer cells are independent of the estrogen receptor. Toxicol Appl Pharmacol 2011; 257: 165-73.

11 Zhang Y, Zhu G, Gu S, Chen X, Hu H, Weng S. Genistein inhibits osteolytic bone metastasis and enhances bone mineral in nude mice. Environ Toxicol Pharmacol 2010; 30: 37-44.

12 Li Y, Bäckesjö CM, Haldosén LA, Lindgren U. Resveratrol inhibits proliferation and promotes apoptosis of osteosarcoma cells. Eur J Pharmacol 2009; 609: 13-8.

13 Park H, Bergeron E, Senta H, Guillemette K, Beauvais S, Blouin R, et al. Sanguinarine induces apoptosis of human osteosarcoma cells through the extrinsic and intrinsic pathways. Biochem Biophys Res Commun 2010; 399: 446-51.

14 Sandur SK, Ichikawa H, Sethi G, Ahn KS, Aggarwal BB. Plumbagin (5-hydroxy-2-methyl-1,4-naphthoquinone) suppresses NF-kB activation and NF-KB-regulated gene products through modulation of p65 and IKB $\alpha$ kinase activation, leading to potentiation of apoptosis induced by cytokine and chemotherapeutic agents. J Biol Chem 2006; 281: 17023-33.

15 Paiva SR, Figueiredo MR, Aragão TV, Kaplan MAC. Antimicrobial activity in vitro of plumbagin isolated from Plumbago species. Mem Inst Oswaldo Cruz 2003; 98: 959-61.

16 Sharma I, Gusain D, Dixit VP. Hypolipidaemic and antiatherosclerotic effects of plumbagin in rabbits. Indian J Physiol Pharmacol 1991; 35: 10-4.

17 Checker R, Sharma D, Sandur SK, Khanam S, Poduval T. Antiinflammatory effects of plumbagin are mediated by inhibition of NFkappaB activation in lymphocytes. Int Immunopharmacol 2009; 9: 949-58.

18 Manu KA, Shanmugam MK, Rajendran P, Li F, Ramachandran L, Hay 
HSIN, et al. Plumbagin inhibits invasion and migration of breast and gastric cancer cells by downregulating the expression of chemokine receptor CXCR4. Mol Cancer 2011; 10: 107.

19 Kuo PL, Hsu YL, Cho CY. Plumbagin induces G2-M arrest and autophagy by inhibiting the AKT/mammalian target of rapamycin pathway in breast cancer cells. Mol Cancer Ther 2006; 5: 3209-21.

20 Aziz MH, Dreckschmidt NE, Verma AK. Plumbagin, a medicinal plant-derived naphthoquinone, is a novel inhibitor of the growth and invasion of hormone-refractory prostate cancer. Cancer Res 2008; 68: 9024-32.

21 Sand JM, Hafeez BB, Jamal MS, Witkowsky O, Siebers EM, Fischer J, et al. Plumbagin (5-hydroxy-2-methyl-1,4-naphthoquinone), isolated from Plumbago zeylanica, inhibits ultraviolet radiation-induced development of squamous cell carcinomas. Carcinogenesis 2012; 33: 184-90.

22 Logothetis $\mathrm{CJ}$, Lin SH. Osteoblasts in prostate cancer metastasis to bone. Nat Rev Cancer 2005; 5: 21-8.

23 Sung B, Oyajobi B, Aggarwal BB. Plumbagin inhibits osteoclastogenesis and reduces human breast cancer-induced osteolytic bone metastasis in mice through suppression of RANKL signaling. Mol Cancer Ther 2012; 11: 350-9.

24 Yoneda T, Williams PJ, Hiraga T, Niewolna M, Nishimura R. A boneseeking clone exhibits different biological properties from the MDAMB-231 parental human breast cancer cells and a brain-seeking clone in vivo and in vitro. J Bone Miner Res 2001; 16: 1486-95.

25 Price JT, Quinn JMW, Sims NA, Vieusseux J, Waldeck K, Docherty SE, et al. The heat shock protein 90 inhibitor, 17-allylamino-17-demethoxygeldanamycin, enhances osteoclast formation and potentiates bone metastasis of a human breast cancer cell line. Cancer Res 2005; 65: 4929-38.

26 Park HH, Lee KY, Kim SH, Lee YJ, Koh SH. L-DOPA-induced neurotoxicity is reduced by the activation of the PI3K signaling pathway. Toxicology 2009; 265: 80-6.
27 Zheng Y, Zhou H, Modzelewski JRK, Kalak R, Blair JM, Seibel MJ, et al. Accelerated bone resorption, due to dietary calcium deficiency, promotes breast cancer tumor growth in bone. Cancer Res 2007; 67: 9542-48.

28 Zheng Y, Zhou H, Brennan K, Blair JM, Modzelewski J, Seibel MJ, et al. Inhibition of bone resorption, rather than direct cytotoxicity, mediates the anti-tumour actions of ibandronate and osteoprotegerin in a murine model of breast cancer bone metastasis. Bone 2007; 40: 471.

29 Jiang Y, Huang Y, Cheng C, Lu W, Zhang Y, Liu X, et al. Combination of thiazolidinedione and hydralazine suppresses proliferation and induces apoptosis by PPARy up-expression in MDA-MB-231 cells. Exp Mol Pathol 2011; 91: 768-74.

30 Ahmad A, Banerjee S, Wang Z, Kong D, Sarkar FH. Plumbagin-induced apoptosis of human breast cancer cells is mediated by inactivation of NF-kB and Bcl-2. J Cell Biochem 2008; 105: 1461-71.

31 Kawiak A, Zawacka-Pankau J, Lojkowska E. Plumbagin induces apoptosis in Her2-overexpressing breast cancer cells through the mitochondrial-mediated pathway. J Nat Prod 2012; 75: 747-51.

32 Aziz MH, Dreckschmidt NE, Verma AK. Plumbagin, a medicinal plant-derived naphthoquinone, is a novel inhibitor of the growth and invasion of hormone-refractory prostate cancer. Cancer Res 2008; 68: 9024-32.

33 Hafeez BB, Zhong W, Mustafa A, Fischer JW, Witkowsky O, Verma AK. Plumbagin inhibits prostate cancer development in TRAMP mice via targeting $\mathrm{PKC}\{$ varepsilon\}, Stat3 and neuroendocrine markers. Carcinogenesis 2012; 33: 2586-92.

34 Devi PU, Solomon F, Sharada A. Plumbagin, a plant naphthoquinone with antitumor and radiomodifying properties. Pharm Biol 1999; 37: 231-6.

35 Li Z, Xiao J, Wu X, Li W, Yang Z, Xie J, et al. Plumbagin inhibits breast tumor bone metastasis and osteolysis by modulating the tumor-bone microenvironment. Curr Mol Med 2012; 12: 967-81. 